\title{
Pausas en la lactancia de bebés y su relación con el origen de la comunicación verbal: un estudio con gemelos dicigóticos
}

\section{Pauses in breastfeeding babies and their relationship with the origin of verbal communication: a study with dizygotic twins}

\author{
Pablo Gomez Ramos ${ }^{1}$ \\ Universidad Autónoma de Madrid
}

Recibido: $19-10-20$

Aceptado: $16-11-20$

Publicado: $21-12-20$

\section{Resumen}

Los bebés humanos son los únicos mamíferos que realizan pausas en el proceso de lactar durante los primeros meses de vida, un hecho único que no se relaciona con ninguna utilidad práctica. En los años 80 del siglo pasado, algunos investigadores relacionaron estas paradas con formatos de intercambio de turnos entre la madre y el hijo dentro de un proceso equivalente a un diálogo o conversación. Utilizando un diseño unifactorial y univariado intrasujeto, hemos realizado observaciones a un par de gemelos dicigóticos intentando comprobar hasta qué punto está generalizada la detención en el proceso de succión y qué papel desempeña la intervención del adulto en dichas conductas. Los resultados muestran, por un lado, que son conductas generalizadas y por otro, que no es necesaria la intervención del adulto, aunque sí las condiciona, lo que reforzaría el carácter innato de las pausas y también su posible relación con el origen del lenguaje.

Palabras clave: Bebés; lactancia; succión; pausas; turnos; lenguaje.

\begin{abstract}
Human babies are the only mammals that pause in the process of breastfeeding during the first months of life, a unique fact that is not related to any practical use. In the 1980s, some researchers linked these pauses to formats of shift exchange between mother and child within a process equivalent to a dialogue or conversation. Using an intrasubject unifactorial and univariate design, we have made observations on a pair of dizygotic twins trying to check how widespread the stop in the sucking process is and what role adult intervention plays in such behaviours. The results show, on the one hand, that these are generalized behaviors and, on the other hand, that the intervention of the adult is not necessary, although it does condition them, which would reinforce the innate character of the pauses and also their possible relation with the origin of the language.
\end{abstract}

Keywords: Babies; breastfeeding; breathing; pauses; shifts; language.

1 Doctor Filosofía y Letras y Graduado en Psicología, Docente en la Universidad Autónoma de Madrid. E-mail: pablogr19@gmail.com ORCID: https://orcid.org/0000-0003-1361-6958

(C) Los autores. Este artículo es publicado por la Revista de Investigación en Psicología de la Facultad de Psicología, Universidad Nacional Mayor de San Marcos. Este es un artículo de acceso abierto, distribuido bajo los términos de la licencia Creative Commons Atribucion - No Comercia_Compartir Igual 4.0 Internacional. (http://creativecommons.org/licenses/by-nc-sa/4.0/) que permite el uso no comercial, distribución y reproducción en cualquier medio, siempre que la obra original sea debidamente citada. 
¿Alguna vez han observado cómo durante las tomas de leche los bebés suelen detenerse cada cierto tiempo? Preguntadas varias madres o bien no lo recuerdan, o bien no han sido conscientes de este hecho. Sin embargo, todas ellas refieren haber seguido procedimientos muy parecidos cuando sus bebés paraban de comer, insistiendo de varias maneras para que retomasen la alimentación, por ejemplo, a través de la palabra, caricias, mimos, mecimientos, movimientos del biberón o de la mama, etc.

El reflejo de succión es un reflejo fisiológico con el que nacemos todos los mamíferos y se relaciona directamente con la supervivencia como forma primera de proceder para conseguir el alimento de la madre. Ahora bien, hay una diferencia esencial entre los animales y el ser humano. La posición de la laringe en los animales es distinta a la nuestra pues la colocación alta en ellos les permitirá durante toda su vida respirar y alimentarse al mismo tiempo, algo que solamente pueden hacer los humanos durante una etapa del desarrollo muy corta. En efecto, las pausas de unos pocos segundos, "innecesarias" para respirar durante la lactancia en humanos, tienen un período temporal muy breve porque aproximadamente a partir de los 6 meses, la laringe desciende desde una posición original más alta $\mathrm{y}$, tras este hecho, las paradas serán totalmente indispensables para respirar, pues ya no podremos nunca más respirar y deglutir al mismo tiempo.

Pero si el análisis topográfico parece claro, más complicado es el análisis funcional de las interrupciones alimenticias de los lactantes o incluso, averiguar su relación con otros reflejos infantiles. Si comparamos la succión y sus pausas asociadas con otros reflejos como los lloros y los gritos de hambre o dolor, observamos algunas diferencias significativas, principalmente en relación con el papel modulador de la madre o del adulto. Todos ellos son reflejos destinados a la supervivencia y, por lo tanto, parecen equiparables funcionalmente, sin embargo, mientras que estas conductas reflejas se producen para captar la atención del adulto, la succión propiamente dicha es independiente a ella.

En otro punto del desarrollo se encuentran parloteos o balbuceos de distinto tipo que no se relacionan ni con la supervivencia ni tampoco con la atención prestada por la madre. En efecto, los bebés balbucean y parlotean muy pronto, y aunque comienza a manifestarse de forma sistemática hacia finales del segundo mes, es posible observar algunas manifestaciones accidentales más precoces incluso antes del primer mes (Le Normand, 2003, p. 32). Gritos y balbuceos han sido registrados y sometidos a análisis espectrográfico, donde se ha observado que son expresiones no sólo tempranas, sino también independientes de la influencia materna o ambiental (Osterrieth y Oléron, 1977, pp. 79-81).

Si nos centramos específicamente en las pausas durante la lactancia, Kaye y Wells (1980) midieron sus tiempos en 52 bebés de ambos sexos y edades comprendidas entre 2 días y 2 semanas obteniendo interesantes resultados, si bien no utilizaron 
sujetos control. Todas las madres, tanto experimentadas como novatas, y tanto con lactancia natural como con lactancia de biberón, respondían de forma instintiva y sistemática a las interrupciones de las tomas mediante mecimientos o zarandeos contingentes (a veces también con caricias o ajustes de la ropa del niño), y tras minuciosos registros temporales apuntaron la hipótesis de que los propios bebés pudieran estar condicionando con el paso del tiempo, las conductas de sus madres. Así, el cese de los zangoloteos resultó ser un mejor desencadenante para la reanudación de la succión que el balanceo en sí mismo, lo que provocaba que la intervención de la madre y sus respectivos mecimientos fuesen cada vez más reducidos, acortándose también las pausas de los bebés (Kaye y Wells, 1980, pp. 38-39).

¿Pero cuál es la función de estas pausas? Aunque ciertamente lo desconocemos, se ha apuntado que la única función obvia es lograr que la madre actuase en un intercambio por turnos con su hijo durante la alimentación y aunque fuese una interacción asimétrica, posiblemente pudiese relacionarse con ritmos parecidos a los que se producen en un diálogo o conversación (Kaye, 1986, pp. 105, 110). La falta de una funcionalidad clara con el proceso de alimentación, condujo a Kaye a vincular estos comportamientos neonatales con un fenómeno de complejas relaciones diádicas que se produce de forma muy temprana entre el bebé y su cuidador y que constituye, posiblemente, el ejemplo más temprano de una actuación por turnos con contingencias o influencias mutuas y que se dan también en juegos cara a cara entre madre e hijo (Kaye, 1986, pp. 56, 112-114).

Asimismo, Bruner (1986) señaló que la adquisición del lenguaje debía contar con unos mecanismos previos que enseñasen al niño la actuación de turnos con intercambios oformatos de interacción, como pautas estandarizadas que se aprenden y reproducen, por ejemplo, en los juegos infantiles. Estos formatos de interacción, propios también de un diálogo, harían posible la actuación por turnos entre niño y el adulto, con roles que con el paso del tiempo serían reversibles (Bruner, 1986, p. 119) y necesarios en la comunicación humana.

Noam Chomsky fue el primero que abogó por un Mecanismo de Adquisición del Lenguaje ( $L A D$ ), una capacidad o estructura mental innata, predispuesta biológicamente y que posibilita la adquisición y generación del lenguaje más allá del refuerzo o de la simple imitación que el niño hace del lenguaje del adulto (Barón y Müller, 2014; Chomsky, 1965). Aunque Bruner (1986, pp. 22, 41) también defendió que nacemos con un Sistema de Apoyo de la Adquisición del Lenguaje, donde se enmarcan las interacciones niño-adulto que se producen durante el juego y que posibilitarán mediante pequeñas proto-conversaciones un aprendizaje ordenado del lenguaje, sí que lo vincula con una ayuda necesaria y transaccional por parte del adulto; incluso las madres ya desde los dos meses de vida de sus hijos, dialogan con ellos como si estos les entendiesen (Bruner, 1986, pp. 69 y ss.). Estas conductas se suceden, por lo tanto, en procesos claramente sociales, lo que rescataría los planteamientos funcionales de Lev Vygotski al 
defender que las conductas se producen en escenarios sociales y no en el vacío (García Madruga y Delgado, 2010, p. 181).

Sin profundizar en disquisiciones sobre si nos encontramos ante procesos propiamente sociales, modulares plenos o interaccionistas de modularidad relativa, lo cierto es que la asunción de un mecanismo innato o genético en la adquisición del habla justificaría no solo la falta de esfuerzo especial con la que aprendemos a hablar sino también que existiesen otras pruebas de facultad primitiva para la comunicación, previas al habla constituida. Entre estos indicios podrían estar, entre otros muchos, la discriminación del bebé hacia el rostro humano (Karmiloff-Smith, 1995, pp. 1298-1299), la imitación volitiva precoz, que se ha explicado también como una especie de diálogo entre bebé y adultos (Killen y Uzgiris, 1981) pero, sobre todo, la capacidad auditiva de percepción de los sonidos del habla humana mediante mecanismos especializados tempranos, incluso prenatales (Le Normand, 2003, pp. 31-32) o también, las conductas de pausa en la lactancia de los bebés.

La relación entre el procesamiento del habla y la conducta de succión se ha comprobado con varios experimentos que demuestran cómo bebés de tan solo 2 meses aumentan el proceso de succión dependiendo de los sonidos de sílabas distintas de la voz humana, distinguiendo incluso cambios en el género de las voces de los hablantes (Jusczyk, Pisoni y Mullennix, 1992). Resultados similares se han observado con imágenes y distintos tipos de sonidos utilizando procedimientos de condicionamiento operante (Rochat, 2004, pp. 90-96).

Las investigaciones de Kaye y Wells (1980) y Kaye (1982), al menos según nuestra información, no crearon escuela ni tuvieron seguimiento en la comunidad científica, por lo que el estado actual de la cuestión es la ausencia de estudios de continuación. Aprovechando la circunstancia de que actualmente estoy trabajando con bebés de pocos meses, he podido analizar en estos dos últimos años, las conductas de interrupción de lactar en muchos bebés, en un número aproximado de 20. La muestra se ha centrado en hermanos gemelos con el fin de comprobar también si son conductas parejas en individuos relacionados genéticamente.

A diferencia de las investigaciones anteriores, aquí se ha utilizado como estímulo discriminativo en vez de mecimientos y/o caricias, la voz, a la cual, como hemos comentado, el ser humano es especialmente sensible ya desde antes del nacimiento y durante los primeros meses de vida. Además, para comparar e intentar dar luz a la idea de la relación entre interrupciones de lactar y el origen de la comunicación o lenguaje preverbal, también se han estudiado a otros tres bebés más, dos de ellos con alteraciones del neurodesarrollo diagnosticadas.

Nuestro objetivo ha sido doble, por un lado, comprobar si efectivamente los bebés pausan de forma generalizada durante la lactancia, pues es importante 
discriminar si se trata de conductas más o menos extendidas o si, por el contrario, son conductas puntuales de unos pocos bebés. Por otro lado, investigar si realmente es necesaria alguna acción reforzadora por parte de la madre o del adulto que los atiende, para que se produzcan y repitan estas conductas. En el caso de que sean conductas generalizadas y de que los bebés no necesiten la intervención de la madre, podrían dar fuerza al carácter filogenético de estos comportamientos y los igualaría con otros reflejos como lloros, gritos, etc. A su vez, el efecto modulador de la voz humana en la expresión conductual de las pausas podría relacionarlas con procesos de preparación para la adquisición posterior del habla.

Dado el número reducido de sujetos analizados, este trabajo tiene un carácter meramente aproximativo a las hipótesis previamente planteadas. No obstante, tras las observaciones realizadas a estos bebés hemos comprobado que efectivamente todos ellos realizan pausas repetidas durante las tomas de leche, y que dichas paradas se producen sin necesidad de actuación ni contestación por parte del adulto.

\section{MÉTODO}

\section{Participantes}

El conjunto muestral estuvo formado por 4 gemelos dicigóticos, siendo todos ellos bebés de baja edad gestacional. Se seleccionaron dos muestras, cada una de ellas compuesta por dos hermanos gemelos. Los hermanos de la muestra 1, de distinto sexo y de 5 meses de edad, nacieron en la semana 28 mientras que los hermanos de la muestra 2, ambos varones y de 3 meses de edad, lo hicieron en la semana 27. Al ser niños pretérminos extremos, las edades corregidas serían aproximadamente de unas 12 semanas menos para cada niño.

Los análisis de la muestra 1 se realizaron en el mes de diciembre de 2017 y enero de 2018 mientras que los de la muestra 2 fueron hechos en marzo y abril de 2018, momento en el que se incorporaron estos hermanos.

Todas las observaciones tuvieron lugar en el mismo nido donde estaban los bebés y en la misma residencia infantil.

Con el fin de contrastar la hipótesis de trabajo y comprobar si se producían también interrupciones en otros bebés, se tomaron medidas, no diarias ni tan sistemáticas, a otros 3 sujetos, que no eran hermanos, 2 de ellos con alteraciones del neurodesarrollo diagnosticadas.

\section{Materiales y equipamiento}

Los materiales empleados en el estudio y con todos los sujetos, fueron biberones con tetinas de tres velocidades y leche Blemil 1. Los datos numéricos y temporales 
se tomaron in situ en cada toma, y eran registrados en una hoja modelo que incluía entre otros apartados, la fecha, nombre del niño, hora de inicio y finalización de la toma, duración total, tiempo total de las pausas y observaciones. Se utilizó una mini grabadora Sony para medir los tiempos de las pausas.

\section{Diseño}

Se estudiaron ambas muestras por separado, ya que llegaron en distintos meses, con sujetos relacionados (hermanos) con un diseño univariado y unifactorial. El diseño podría relacionarse análogamente con estudios de caso único o incluso, intrasujeto, ya que los hermanos estudiados comparten no sólo material genético sino también características ambientales idénticas, al estar en un mismo tiempo y lugar.

Únicamente se consideró una variable independiente a manipular y que fue la voz del experimentador, cuya influencia se comprobó a su vez en una variable dependiente, la interrupción en el reflejo de succión durante la conducta de lactar.

Los sujetos fueron elegidos aleatoriamente para cada una de las condiciones experimentales. En la muestra 1 el niño actuó como sujeto experimental mientras que su hermana lo hizo en la condición de control. En la muestra 2 un niño actuó como sujeto experimental y su hermano lo hizo en la condición de control (véase Figura 1).
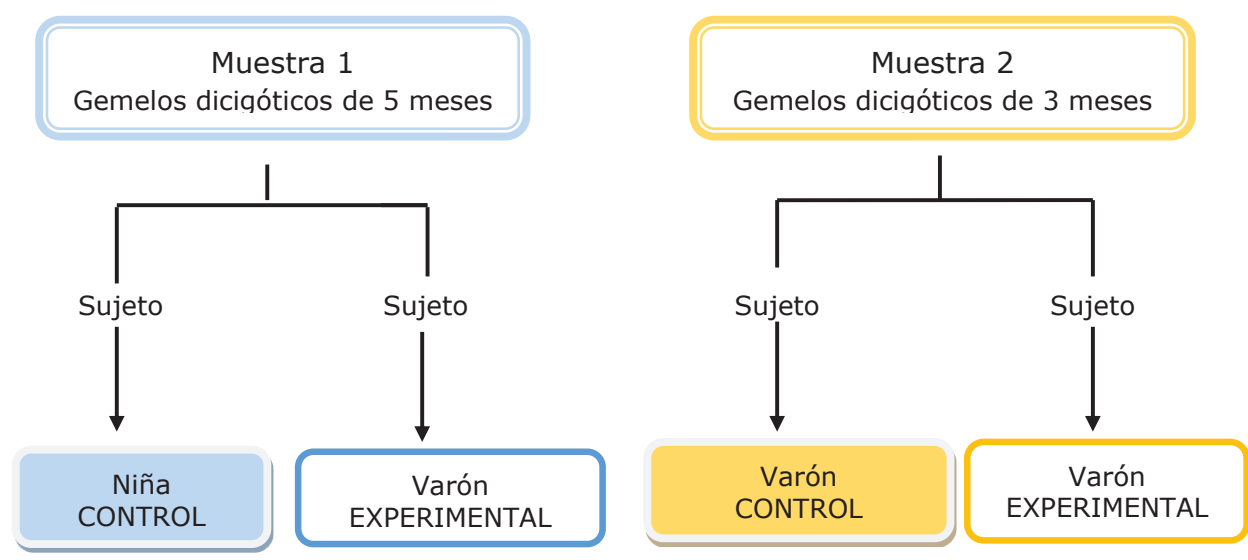

Figura 1. Diagrama de flujo de las condiciones experimentales de los sujetos

\section{Procedimiento}

La observación conductual se realizó de forma individual y separada con cada uno de los sujetos objeto de estudio. En todos los ensayos se daba el biberón al bebé mientras se registraba simultáneamente el número de pausas y el tiempo que duraba cada una de ellas. 
El experimentador observaba tres veces por noche y durante dos meses, las conductas de lactar e iba registrando las pausas que realizaban los sujetos asignados a una de las dos condiciones, control o experimental. En el caso de los sujetos control sin intervención alguna, no se hablaba ni reforzaba la conducta, $\mathrm{y}$ los datos se iban apuntando según se iban produciendo mientras que con los sujetos experimentales se introducía como intervención la voz del experimentador, que actuaba en este caso como variable independiente o causal y que consistía en contar numéricamente a partir de 1 . Tras varias reflexiones optamos porque la voz empleada tuviese la menor variación posible en sus componentes paralingüísticos (entonación, prosodia, etc.).

Además de la utilización de hermanos gemelos, se ha intentado mostrar igualdad en otras muchas circunstancias con el fin de poder controlar la influencia de posibles variables extrañas; así, por ejemplo, todos los bebés eran manipulados por la misma persona, todas las tomas se realizaban por la noche, con tetinas idénticas y cada sujeto partía de la misma cantidad de leche.

\section{RESULTADOS}

Tal y como señalamos en el apartado de diseño, los resultados vienen en gran medida condicionados por las hipótesis de trabajo y por la metodología utilizada. Nuestro primer objetivo fue comprobar si los bebés pausan o no durante la lactancia y, en segundo lugar, si la intervención del adulto es necesaria o influye en las pausas. Para ello se ha hecho un análisis estadístico ${ }^{2}$ que tiene solamente un valor orientativo ya que el número de participantes es pequeño. No obstante, los resultados de la muestra 1 pudieron completarse con la aparición inesperada, meses después, de otro par de gemelos.

Muestra 1. El número de pausas en 17 ensayos fue el siguiente: 489 pausas en el sujeto control mientas que el sujeto experimental realizó un total de 426. A tenor de los datos del sujeto control queda comprobada la hipótesis de trabajo según la cual los dos bebés hacen pausas independientemente de la acción del adulto.

Para completar estos resultados y poder observar si existen diferencias estadísticamente significativas en el número de pausas de ambos participantes, se aplicó como estadístico de contraste la $t$ de Student ya que desconocemos la varianza poblacional. El resultado en la muestra 1 fueron diferencias marginalmente significativas, con una significación bilateral del $\mathbf{0 . 0 5 3}$ que indicaría un posible rechazo de la hipótesis nula de igualdad de medias. No obstante, aquí los datos del sujeto experimental podrían estar algo infra-estimados por la intervención del adulto, como explicaremos más adelante. Véanse los datos completos en la Tabla 1.

2 Mi agradecimiento al Dr. D. José Manuel Reales, de la Facultad de Psicología de la UNED, por su ayuda en la elaboración de los datos estadísticos. 
Tabla 1

Datos estadísticos de la muestra 1

Estadísticas de muestras emparejadas

\begin{tabular}{|l|c|c|c|c|}
\cline { 2 - 5 } \multicolumn{1}{c|}{} & Media & N (ensayos) & Desviación & Desv. Error promedio \\
\hline Número de pausas. Sujeto control & 28,76 & 17 & 8,31 & 2,01 \\
\hline Número de pausas. Sujeto experimental & 25,05 & 17 & 9,07 & 2,2 \\
\hline
\end{tabular}

Correlaciones de muestras emparejadas

\begin{tabular}{|l|c|c|c|}
\cline { 2 - 4 } \multicolumn{1}{c|}{} & N (ensayos) & Correlación & Significación \\
\hline Número de pausas & 17 & 0,65 & 0,005 \\
\hline
\end{tabular}

Prueba de muestras emparejadas. Diferencias emparejadas

\begin{tabular}{|l|c|c|c|c|}
\cline { 2 - 5 } \multicolumn{1}{c|}{} & Media & Desviación & Desv. Error promedio & $\mathbf{9 5 \%}$ interv. Inf. \\
\hline Número de pausas & 3,7 & 7,3 & 1,77 & $-0,049$ \\
\hline
\end{tabular}

Prueba de muestras emparejadas. Diferencias emparejadas

\begin{tabular}{|l|c|c|c|c|}
\cline { 2 - 5 } \multicolumn{1}{c|}{} & $\mathbf{9 5 \%}$ intervalo superior & $\mathbf{t}$ & gl & Sig bilateral \\
\hline Número de pausas & 7,46 & 2,09 & 16 & 0,053 \\
\hline
\end{tabular}

Muestra 2. Tal y como señalamos más arriba, dos meses después de analizar la muestra 1, tuvimos ocasión de observar a otro par de gemelos dicigóticos, en este caso 2 varones e igualmente pretérminos extremos. El número de pausas en 14 ensayos fue el siguiente: 447 pausas en el sujeto control mientas que el sujeto experimental realizó un total de 472 . Como en el caso anterior quedó comprobada la hipótesis de las interrupciones en los bebés durante la lactancia y también se observó, al igual que en la muestra anterior, pausas en el sujeto control independientes de la conducta del adulto. En esta muestra también se aplicó como estadístico de contraste la $t$ de Student. En este caso no se observan diferencias estadísticamente significativas, con una significación bilateral del $\mathbf{0 . 4 8 1}$ que indicaría la aceptación de la hipótesis nula de igualdad de medias entre los dos sujetos. Los resultados estadísticos aparecen en la Tabla 2.

Además de estas muestras tuvimos ocasión de observar a otros 3 bebés para comprobar si también hacían pausas durante las tomas de alimentación. En todos los ensayos el procedimiento seguido fue el mismo que en los ejemplos anteriores, aunque aquí no se hablaba durante ninguna de las tomas para poner a prueba la hipótesis de la posible conducta innata e independiente de la intervención adulta. Cabe destacar que estos sujetos se observaron de manera menos sistemática y numerosa que con las muestras 1 y 2 . 
Tabla 2

Datos estadísticos de la muestra 2

Estadisticas de muestras emparejadas

\begin{tabular}{|l|c|c|c|c|}
\cline { 2 - 5 } \multicolumn{1}{c|}{} & Media & N (ensayos) & Desviación & Desv. Error promedio \\
\hline Número de pausas. Sujeto control & 31,92 & 14 & 17,02 & 4,55 \\
\hline Número de pausas. Sujeto experimental & 33,71 & 14 & 17,01 & 4,54 \\
\hline
\end{tabular}

Correlaciones de muestras emparejadas

\begin{tabular}{|l|c|c|c|}
\cline { 2 - 4 } \multicolumn{1}{c|}{} & N (ensayos) & Correlación & Significación \\
\hline Número de pausas & 14 & 0,85 & 0,001 \\
\hline
\end{tabular}

Prueba de muestras emparejadas. Diferencias emparejadas

\begin{tabular}{|l|c|c|c|c|}
\cline { 2 - 5 } \multicolumn{1}{c|}{} & Media & Desviación & Desv. Error promedio & 95\% interv. Inf. \\
\hline Número de pausas & $-1,78$ & 9,2 & 2,46 & $-7,10$ \\
\hline
\end{tabular}

Prueba de muestras emparejadas. Diferencias emparejadas

\begin{tabular}{|l|c|c|c|c|}
\cline { 2 - 5 } \multicolumn{1}{c|}{} & $\mathbf{9 5 \%}$ intervalo superior & $\mathbf{t}$ & gl & Sig bilateral \\
\hline Número de pausas & 3,53 & $-0,72$ & 13 & 0,481 \\
\hline
\end{tabular}

Muestra 3. En este caso se trataba de un bebé varón prácticamente recién nacido, de tan solo 7 días. Las observaciones, que no fueron sistemáticas ni diarias, empezaron el día 18 de enero de 2018 y terminaron el 20 de febrero del mismo año. Aunque en el primer ensayo se constató un total de 15 pausas, que durante los 8 minutos que duró la toma, sumó un cómputo de 44 segundos, se observó una evolución hacia mayor número de interrupciones que llegó a ascender el último día de ensayo hasta las 70 en una toma de 15 minutos.

Muestra 4. Esta participante era una niña diagnosticada con síndrome de Williams $^{3}$. La primera prueba fue el 10 de febrero de 2018 a la edad de 21 días y realizó 12 pausas en 15 minutos de toma con un total de 40 segundos. La última observación se realizó el 16 de mayo. A diferencia de la muestra 3, aquí no hubo progresión a un mayor número de pausas, y se mantuvo más o menos constante con una media de 7 pausas por toma.

Muestra 5. En enero de 2020 tuvimos ocasión de observar a un bebé con una encefalopatía congénita y daño permanente en ambos hemisferios cerebrales. Debido a esto, las interacciones fueron muy complicadas ya que el niño no respondía a un ciclo normalizado de alimentación. Aunque no pudimos comprobar las conductas de

3 En relación con la comunicación y adquisición del habla, aunque los individuos con síndrome de Williams suelen presentar déficits intelectuales, tienen sin embargo preservadas las capacidades lingüísticas generales (Karmiloff-Smith et al., 1995). 
pausa, pues había limitaciones claras de la motricidad bucal, con afectación de los automatismos de succión, sí observamos que mostraba una atención clara y preferente al habla de los adultos, con movimientos y orientaciones de cabeza.

Importa añadir que el estudio longitudinal que hemos llevado a cabo se basa en el número de pausas realizadas por los sujetos de estudio, mientras que Kaye y Wells (1980) manejaron principalmente los segundos de duración tras las pausas o desde el final de los mecimientos, llegando a la conclusión de que la duración media de la intervención de una madre promedio disminuyó de los 3,1 segundos a los dos días, a los 1,8 segundos en la segunda semana (Kaye y Wells, 1980, p. 39). En nuestro caso, el número de pausas no sólo no decreció, sino que fue en claro aumento, pasando de 16 a 28 pausas en el Experimento n. 1 y de 28 a 49 pausas en el Experimento n. ${ }^{\circ}$ 2. Más aún, si la comparación se realiza teniendo en cuenta los tiempos de intervención del adulto con los sujetos experimentales, de forma análoga a como hicieron Kaye y Wells, la propensión decreciente señalada por estos investigadores tampoco fue constatada, sino todo lo contrario; así por ejemplo, la Figura 2 muestra una tendencia al alza ya que en el Experimento n. ${ }^{\circ} 1$, el tiempo de intervención del experimentador pasó de 2,1 segundos a 3,6 segundos mientras que en el Experimento n. ${ }^{\circ} 2$ el tiempo promedio pasó de 3,5 a 4 segundos.

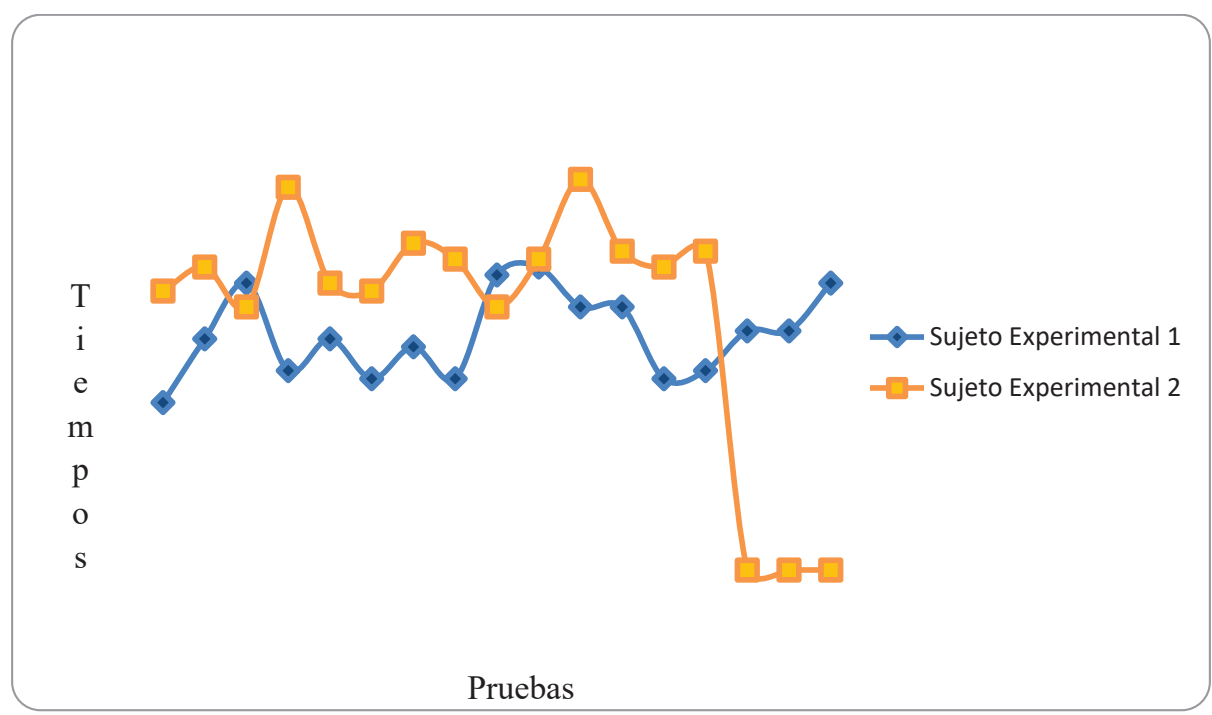

Figura 2. Segundos de intervención del experimentador

Más coincidentes son las medias del tiempo de las pausas, que Kaye y Wells (1980, p. 29) cifran entre los 4 y 15 segundos y que nosotros hemos comprobado entre los 2 y 12 segundos. No obstante, cabe advertir que el número de muestras 
que hemos analizado fue muy inferior y que la variable independiente utilizada fue la voz y no el movimiento o zarandeo.

\section{DISCUSIÓN}

Las hipótesis planteadas en este trabajo se han visto confirmadas por los resultados obtenidos. Por un lado, se ha comprobado que todos los bebés realizan pausas repetidas durante las tomas de alimentación, y por otro, que no fue necesaria la intervención del cuidador para que se desarrollasen dichas conductas. A diferencia de los experimentos de Kaye y Wells (1980) nosotros no manejamos los mecimientos como variable independiente, sino la voz, y sí utilizamos sujetos control para ver hasta qué punto era necesaria o no la intervención del adulto.

Como vimos en el apartado anterior, en el Experimento n. ${ }^{\circ}$ 1, el sujeto control, que no fue reforzado, realizó mayor número de paradas que su hermano gemelo, con un resultado de 0,053 en el estadístico de contraste $T$, un dato de igualdad marginalmente significativo que podría indicar un rechazo de la hipótesis nula de igualdad de medias a favor del sujeto control.

Sin embargo, en el Experimento n. 2 el resultado de la prueba no fue estadísticamente significativo, con una significación bilateral del 0,481 , por lo que la hipótesis nula se mantiene, es decir, ambos gemelos hicieron pausas independientemente de la conducta del adulto y sin diferencias estadísticamente significativas. En este caso, el sujeto control realizó 25 paradas menos que su hermano experimental, aunque los datos recogidos para esta prueba incluyeron 3 ensayos menos que en el experimento 1, por lo que no sabemos si con el mismo número de ensayos en ambos experimentos los datos hubiesen estado más cerca o más lejos de los obtenidos.

Si analizamos el número de pausas por cada ensayo encontramos algunos resultados interesantes. En concreto, las pausas en un mismo ensayo son a veces prácticamente las mismas entre hermanos gemelos, lo que podría ser un elemento más que refuerce el carácter, no solamente innato, sino también genético de estas conductas; sí un hermano en un ensayo hacía muchas pausas, su hermano en ese mismo ensayo también, o viceversa, si el hermano tiene escaso número de pausas, el otro hermano replicó también el mismo comportamiento. Así por ejemplo, en el Experimento 1, comparando el número de pausas del sujeto control con el número de pausas del sujeto experimental, tenemos que en el ensayo n. ${ }^{\circ} 2$ fueron 17 y 16 respectivamente, en el ensayo n. 3 , fueron 16 y 15 o en el ensayo n. 4 fueron iguales, 17 y $17^{4}$. En el Experimento 2, vemos este mismo fenómeno en

4 Se dieron también otros resultados singulares. Por ejemplo, el día 1 de abril de 2018, volvimos a observar a los hermanos del Experimento 1 y aunque el sujeto experimental estaba resfriado y tomó la mitad de la leche, hizo un número de 12 pausas; su hermana control, aunque no estaba congestionada también hizo un número de pausas prácticamente igual: 13 . 
varios ensayos; en el ensayo n. ${ }^{\circ} 1$, el sujeto control realizó 24 paradas frente 26 del sujeto experimental, en el ensayo n. ${ }^{\circ} 3$ el número fue el mismo, 54 frente a 54 , y lo mismo ocurrió en el ensayo n..$^{\circ} 10$, con 18 y 18 pausas, o en el ensayo n. $^{\circ} 13$ donde el sujeto control hizo 12 pausas frente al sujeto experimental que realizó 10 paradas. En todo caso, los sujetos control prueban conductas de pausa que no pueden relacionarse con aprendizajes ni con condicionamientos previos, y sí con un alto grado de automatismo.

¿Pero cómo interpretar estos y otros resultados? Somos conscientes de las debilidades metodológicas, de la escasez de sujetos analizados y también de la posibilidad de variables extrañas no controladas en el estudio, por lo que los datos numéricos deben considerarse simplemente como una aproximación a la generalidad del proceso estudiado. No obstante, podemos apuntar algunas reflexiones que podrían completar y dar una nueva visión a los estudios de los años 80. Por un lado, al igual que constataron entre otros, Kaye y Wells, parece claro que los bebés participan en un proceso innato de paradas al lactar cuyo significado está lejos de ser descifrado. Estos autores concluyeron, con los datos obtenidos, que las pausas de los bebés de 2 semanas son más cortas que las que realizaban los mismos niños a la edad de 2 días, ya que las madres van aprendiendo que los bebés retoman la succión más que con los mecimientos en sí, con la parada de los mismos (Kaye y Wells, 1980, pp. 42-44), algo que nosotros no hemos observado. Los tiempos de intervención del adulto, el número de pausas y los tiempos de las mismas, fueron en ascenso conforme pasaron las semanas, aumentando la "conversación" en la díada. Por lo tanto, el papel secundario de la madre respecto a las actuaciones de los bebés que defendían estos investigadores, no se ha observado con tanta claridad en el presente estudio; los bebés, bastante después de la segunda semana, seguían haciendo pausas, a veces muy largas, si bien hay que tener en cuenta que los niños eran tratados por un mayor número de personas y no sólo por un único cuidador, por lo que siguiendo el razonamiento de estos autores, los bebés pudieron no haber "enseñado" adecuadamente al adulto la forma de reducir las interacciones.

Sin embargo, y aunque la tarea del cuidador no parece necesaria en la expresión de las pausas, sí podría ser más activa de lo defendido hasta ahora si se decidiese intervenir en las conductas de parada, a tenor de los resultados obtenidos con los sujetos experimentales. En la condición experimental, una vez que un niño iniciaba la pausa, empezábamos a contar, y nos deteníamos en cuanto el bebé reiniciaba nuevamente la succión de leche. Obsérvese que aunque la topografía es distinta, la función contingente entre la interrupción del habla del experimentador y la reanudación de la succión, podría ser equivalente a lo que ocurre cuando las madres detienen los mecimientos y los bebés reanudan la toma del alimento como observaron Kaye y Wells (1980).

No obstante, el mayor protagonismo del adulto vino al detectarse, al principio por pura casualidad, que las pausas y la reanudación de las mismas, podían 
ser moduladas fácilmente mediante la voz. Aunque nuestra intención fue utilizar una variable independiente que estuviese involucrada de manera más directa en la comunicación conversacional que los simples zarandeos, como es el sonido humano, y comprobar si un estímulo hablado tenía importancia o no, ya señalamos que la voz se limitó a contar números, de forma monótona, a partir del 1. Quisimos de este modo despojar al estímulo hablado de cualquier elemento paralingüístico que pudiese parecerse a la maternés, habla que utilizan las madres al dirigirse a sus bebés. Aún así, constatamos que los bebés de la condición experimental, cuando estaban pausando, reiniciaban la succión de forma casi inmediata si el experimentador decidía parar de contar. O al contrario, mientras succionaban, el experimentador podía detener el proceso poniéndose simplemente a hablar o numerar de nuevo. En estos cambios de conducta del bebé mediaban solamente $1 \mathrm{o}$ 2 segundos de tiempo.

La atención intencional de los bebés hacia la voz humana parece ser la clave. Se ha comprobado que ya desde el periodo fetal, los bebés son capaces de discriminar sonidos, llegando a adoptar comportamientos de succión distintos según los sonidos que perciben y reaccionando selectivamente a la voz y palabras humanas (Le Normand, 2003, pp. 31-32). Más aún, al parecer a partir de la tercera semana son capaces de discriminar la variabilidad de distintas voces, mostrando preferencia por las voces femeninas en general, y por la voz de la madre en particular (Aguado, 2003, pp. 48-49), y a los 2 meses distinguen hasta sutiles variaciones fonéticas en las sílabas que oyen, con una percepción categórica similar a la que hacen los adultos (Jusczyk, Pisoni y Mullennix, 1992).

No podemos obviar, sin embargo, que la intervención consciente del adulto podría hacer que se contabilizasen, en los sujetos de la condición experimental, un número total de pausas dispar, y que por lo tanto, con respecto a los sujetos control, hubiese también variaciones. Dicho de otro modo, la participación del adulto implicaría romper cualquier patrón de aleatoriedad presente hasta ese momento. Pero aunque el procedimiento de condicionar las pausas según la intervención (o el antojo) del adulto podría indicar una contaminación de los datos estadísticos, e incluso invalidarlos por manipulación del experimentador, (aunque finalmente estos datos no fueron incluidos en el cómputo de las pausas), creemos que por el contrario viene a reforzar, si cabe con más fuerza, la idea de que adulto y bebé forman una díada de interacciones bastante parecida a la que se da en el intercambio de turnos de una conversación, tal y como defendieron Kaye o Bruner ${ }^{5}$.

5 La actuación por turnos parece darse no solo entre madre e hijo, sino también entre los propios bebés. Hemos observado como existe una interrelación entre ellos a la hora de demandar el alimento cuando comparten un grupo. Así, por ejemplo, casi nunca lloran a la vez, y pueden parar el llanto para dejar paso al lloro de otro compañero, en un proceso de interrelaciones donde parecieran entender quién va primero o quién tiene más necesidad de alimento. En el caso de los gemelos dicigóticos comprobamos como durante la toma, parecen incluso reconocer el llanto de su propio hermano pues paran las succiones y lo escuchan atentamente durante unos segundos antes de continuar, algo que no ocurre cuando oyen llorar a otro bebé. 
Ahora bien, para poder relacionar las pausas con interrelaciones comunicativas, deben descartarse primero otros posibles significados en el origen de las mismas. No existen, o no se han encontrado, razones de utilidad práctica para las interrupciones de los bebés durante la lactancia; no parecen vinculadas a la respiración, a la deglución ni al descanso (Kaye, 1986, p. 56). Podrían relacionarse con procesos de imitación que se dan en los niños ya desde las primeras semanas de vida (Meltzoff y Moore, 1992). También los cambios observados en las mismas succiones se han relacionado como un modo para poder oír mejor determinados sonidos, en particular la voz de su madre (DeCasper y Fifer, 1980) o incluso, para enfocar y obtener imágenes menos borrosas (Bruner, 1986, p. 25). Sin embargo, ninguna de estas explicaciones alternativas encaja satisfactoriamente en nuestro caso, ya que las conductas de los sujetos control evidenciaron que los bebés no necesitan del modelo adulto, ni oír la voz de su cuidador, y ni siquiera, tener los ojos abiertos, para realizar las pausas en la alimentación.

Igualmente, la falta de estímulos discriminativos y de los refuerzos contingentes en los sujetos control, dificulta también explicaciones enmarcadas en un sistema de aprendizaje por condicionamiento operante. El niño detiene muchas de sus actividades ante la voz humana, lo que explicaría que los sujetos experimentales cesen momentáneamente en su actividad de mamar, pero no explica el porqué los sujetos control, ante la ausencia de cualquier voz, realizan también esas mismas pausas. Los bebés pausan independientemente de si se les habla o se les mece, por lo que la cadena estímulo-respuesta-consecuencia no parece operar de forma tan clara en el estudio que hemos realizado, lo que reforzaría la equivalencia de las pausas con otros reflejos innatos.

Debemos reconocer que el número de muestras analizado es escaso, ya que se trata prácticamente de 2 diseños de caso único, por lo que de cara a futuras líneas de investigación sería necesario una muestra mayor y suficientemente representativa, para evaluar sí el habla adulta, modifica o no las pausas de los lactantes y en qué medida y circunstancias lo hace. Utilizar, por ejemplo, un análisis de series temporales con medidas repetidas pre-test y post-test, clarificaría el efecto causal de la variable independiente en cada sujeto concreto y no sólo en su equivalente genético. También pensamos que sería relevante contar con muestras específicas de sujetos con problemas de comunicación y lenguaje. Ya señalamos que si bien uno de los bebés tenía un diagnóstico de síndrome de Williams, realizaba del mismo modo pausas durante la succión de las tomas, y como este trastorno no es especialmente llamativo en cuanto alteraciones del lenguaje, sí sería interesante tener estudios longitudinales de niños con alteraciones posteriores en el habla (trastornos del espectro autista, trastornos específicos del lenguaje, etc.) para poder relacionar las pausas y el desarrollo ulterior del lenguaje.

Lo que parece haber quedado claro, de momento, y que viene a confirmar estudios anteriores, es que los bebés durante los primeros meses de vida, y sin 
necesidad de experiencia previa, hacen pausas durante el proceso de lactancia. Sin embargo, lo que no se había señalado hasta ahora es que estas pausas parecen seguir un patrón común para el cual no es necesaria acción alguna por parte del adulto. Los bebés no participan en procesos de imitación y no necesitan reforzamiento, ni acción hablada o táctil del cuidador para realizar y continuar con el proceso de paradas, incluso cabría decir que vienen programados con ellas, si bien es cierto, que son sensibles a la intervención externa, especialmente a la voz humana. Si estas pausas son competencias básicas y definitorias de un módulo innato facilitador de la adquisición posterior del lenguaje verbal humano, es algo que aún está por dilucidar.

\section{Fuentes de financiamiento / Funding:}

Financiado autónomamente.

\section{Rol de los autores / Authors Roles:}

PGR: Autor de la investigación y el artículo.

\section{Aspectos éticos / legales; Ethics / legals:}

Se han cumplido los estándares eticos europeos propios de investigaciones con humanos y niños.

\section{Conflicto de intereses / Competing interests:}

Declaro no haber encontrado conflictos de interés al realizar el presente informe.

\section{REFERENCIAS}

Aguado, G. (2003). Dimensiones perceptiva, social, funcional y comunicativa del desarrollo. En J. Narbona y Cl. Chevrie-Muller (eds.), El lenguaje del niño. Desarrollo normal, evaluación y trastornos (pp. 47-61). $2^{\mathrm{a}}$ ed. Masson.

Barón, L. y Müller, O. (2014). La teoría lingüística de Noam Chomsky: del inicio a la actualidad. Lenguaje, 42 (2), 417-442. http://www.scielo.org.co/pdf/leng/v42n2/ v42n2a08.pdf

Bruner, J. S. (1983). Child's talk: learning to use language. Oxford University Press. [Trad. cast.: El habla del niño. Paidós, 1986].

Chomsky, N. (1965). Aspects of the theory of syntax. Massachusetts Institute of Technology Press [Trad. cast.: Aspectos de la teoría de la sintaxis. Aguilar, 1971].

DeCasper, A. y Fifer, W. (1980). Of human bonding: newborns prefer their mothers' voices. Science, 208, (4448), 1174-1176. http://bernard.pitzer.edu/ dmoore/psych199s03articles/of_human_bonding.pdf

García Madruga, J.A. y Delgado, B. (2010). La adquisición del lenguaje: relación con la comunicación y el pensamiento. En J. A. García Madruga y J. Delval (coords.), 
Psicología del desarrollo I (pp. 171-205). Universidad Nacional de Educación a Distancia.

Jusczyk, P.W., Pisoni, D.B. y Mullennix, J. (1992). Some consequences of stimulus variability on speech processing by 2-month-old infants. Cognition, 43(3), 253-291. https://doi.org/10.1016/0010-0277(92)90014-9

Karmiloff-Smith, A. (1995). Annotation: The extraordinary cognitive journey from foetus through infancy. Journal of Child Psychology and Psychiatry, 36, 1293-1313. http:// www.academia.edu/download/45448756/Annotation_The_Extraordinary_Cognitive_J20160508-1059-1fq9w7.pdf

Karmiloff-Smith, A., Klima, E., Bellugi, U., Grant, J. y Baron-Cohen, S. (1995). Is There a Social Module? Language, Face Processing, and Theory of Mind in Individuals with Williams Syndrome. Journal of Cognitive Neuroscience, 7 (2), 196-208. https:// www.mitpressjournals.org/doi/abs/10.1162/jocn.1995.7.2.196

Kaye, K. y Wells, A. (1980). Mothers' jiggling and the burst-pause pattern in neonatal feeding. Infant Behavior and Development, 3, 29-46. https://doi.org/10.1016/S01636383(80)80005-1

Kaye, K. (1982). The mental and social lifes of babies: How parents create persons. University of Chicago Press [Trad. cast.: La vida mental y social del bebé: cómo los padres crean personas. Paidós, 1986].

Killen, M. y Uzgiris, I. (1981). Imitation of actions with objects: the role social meaning. The Journal of Genetic Psychology, 138 (2), 219-229. https://doi.org/10.1080/002213 25.1981.10534136

Le Normand, M.T. (2003). Modelos psicolingüísticos del desarrollo del lenguaje. En J. Narbona y Cl. Chevrie-Muller (eds.), El lenguaje del niño. Desarrollo normal, evaluación y trastornos (pp. 29-45). $2^{\mathrm{a}}$ ed. Masson.

Melzoff, A. y Moore, K. (1992). Early Imitation Within a Functional Framework: The Importance of Person Identity, Movement, and Development. Infant Behav Dev. 15 (4), 479-505. https://www.ncbi.nlm.nih.gov/pmc/articles/PMC4137790/

Osterrieth, P. A. y Oléron, P. (1977). Los modos de expresión. En H. Gratiot-Alphandéry y R. Zazzo (eds.), Tratado de Psicología del niño, vol. VI. Ediciones Morata.

Rochat, Ph. (2004). El mundo del bebé (Serie Bruner).Ediciones Morata. 